\title{
The repertoire of maternal anti-viral antibodies in human newborns
}

\author{
Christian Pou 1,4, Dieudonné Nkulikiyimfura ${ }^{1,4}$, Ewa Henckel2,3, Axel Olin ${ }^{1}{ }^{1}$, Tadepally Lakshmikanth', \\ Jaromir Mikes', Jun Wang1, Yang Chen', Anna Karin Bernhardsson 1,3, Anna Gustafsson, ${ }^{2,3}$, \\ Kajsa Bohlin ${ }^{2,3}$ and Petter Brodin (1) ${ }^{1,3 \star}$
}

\begin{abstract}
All circulating immunoglobulin G (IgG) antibodies in human newborns are of maternal origin ${ }^{1}$ and transferred across the placenta to provide passive immunity until newborn IgG production takes over 15 weeks after birth². However, maternal IgG can also negatively interfere with newborn vaccine responses $^{3}$. The concentration of IgG increases sharply during the third trimester of gestation and children delivered extremely preterm are believed to largely lack this passive immunity ${ }^{1,2,4}$. Antibodies to individual viruses have been reported $^{5-12}$, but the global repertoire of maternal IgG, its variation in children, and the epitopes targeted are poorly understood. Here, we assess antibodies against 93,904 epitopes from 206 viruses in 32 preterm and 46 term motherchild dyads. We find that extremely preterm children receive comparable repertoires of IgG as term children, albeit at lower absolute concentrations and consequent shorter half-life. Neutralization of the clinically important respiratory syncytial virus (RS-virus) was also comparable until three months of age. These findings have implications for understanding infectious disease susceptibility, vaccine development, and vaccine scheduling in newborn children.
\end{abstract}

Antibodies provide important protection against infectious diseases; individuals with inborn errors of immunity involving IgG production typically present with severe infections early in life ${ }^{13}$. Such individuals also often suffer from inflammatory conditions and autoimmunity, illustrating the immunomodulatory roles played by IgG antibodies ${ }^{14}$. Newborn children cannot produce IgG antibodies immediately after birth; instead, they rely on passive immunity from maternal IgG ${ }^{15}$. To gain a deeper insight into the repertoire of maternal antibodies actively transferred to newborn children during pregnancy, we took advantage of a recently developed viral epitope scanning method (VirScan) ${ }^{16}$, where bacteriophages present 56-amino-acid-long linear peptides that overlap by 28 amino acids to collectively encompass the entire genomes of 1,276 viral strains from 206 viral species known to infect human cells (Fig. 1a). This method works by incubating plasma samples normalized to total IgG concentration with the phage library to form IgGphage immunocomplexes, which are captured by magnetic beads. Immunoprecipitated bacteriophages then undergo lysis and are sequenced to identify the IgG-targeted epitopes (Fig. 1a). VirScore is the output given by VirScan and corresponds to the number of peptide hits that do not share epitopes. VirScore is incremented by 1 when a hit is enriched in the 'output' compared with the 'input' (Methods). Besides removing hits occurring as a consequence of unspecific binding to beads only, it is also important to identify cross-reactive antibodies. This is done by ignoring hits that share a subsequence of at least seven amino acids with any other enriched hit found in the same sample. We applied this method to 78 motherchild dyads from a recently reported birth cohort at the Karolinska University Hospital in Stockholm ${ }^{17}$, with samples collected at birth (cord blood) and weeks 1, 4, and 12 after birth (Fig. 1b). According to $\mathrm{Xu}$ et al. ${ }^{16}$, adult individuals were seropositive for 5-10 different viruses ${ }^{16}$; a similar result was found for the parents in our birth cohort (Fig. 1c). The most frequently targeted viruses in parents are cytomegalovirus (CMV), Epstein-Barr virus (EBV), herpes simplex virus type 1 (HSV-1), and rhinovirus A (Extended Data Fig. 1a,b). Our cohort consisted of 32 extremely preterm children born before 30 weeks of gestation, and 46 term children ( $>37$ weeks of gestation); we applied VirScan to analyze the global repertoires of maternal antibodies to viruses present at birth, in all of these children. We found that preterm and term children have very similar repertoires of maternal IgG, irrespective of gestational age at birth (Fig. 1d). To further investigate this, we performed a principal component analysis based on the global IgG repertoires for each child at birth or during week 1, but found no segregation between preterm and term children (Extended Data Fig. 2). This was unexpected given previous reports suggesting that IgG transfer occurs primarily during the final trimester, leaving preterm children largely unprotected ${ }^{2}$. Even the most extremely preterm children in our cohort, born at 24 weeks of gestation, had an antiviral IgG repertoire comparable to that found in term-delivered children (Fig.1d). Similar to their parents, the most frequently targeted viruses in newborn children were adenovirus C, CMV, EBV, HSV-1, and rhinovirus A (Fig. 1d).

After describing the viral species targeted by passive immunity in preterm and term infants, we were curious to investigate the individual epitopes targeted in each virus, since this is an important determinant of protective immunity. The number of epitopes targeted by maternal antibodies in newborn children correlate with viral genome size and, consequently, the total number of peptides required to cover the genome of a particular virus (Fig. 2a). Certain epitopes are more often targeted during an immune response than others, so we wanted to know whether antibodies to such immunodominant epitopes were present in the IgG repertoire of newborns. As an example, we show antibodies targeting the major surface glycoprotein G, an important RS-virus protein that contains an immunodominant epitope in its 141-224 amino acid region. We noted that antibodies specific to this epitope were frequently targeted in mothers; these antibodies were also transferred from mothers to their

'Science for Life Laboratory, Department of Women's and Children's Health, Karolinska Institutet, Stockholm, Sweden. ${ }^{2}$ Department of Clinical Science, Intervention and Technology, Karolinska Institutet, Stockholm, Sweden. ${ }^{3}$ Department of Newborn Medicine, Karolinska University Hospital, Stockholm, Sweden. ${ }^{4}$ These authors contributed equally: Christian Pou, Dieudonné Nkulikiyimfura. *e-mail: petter.brodin@ki.se 


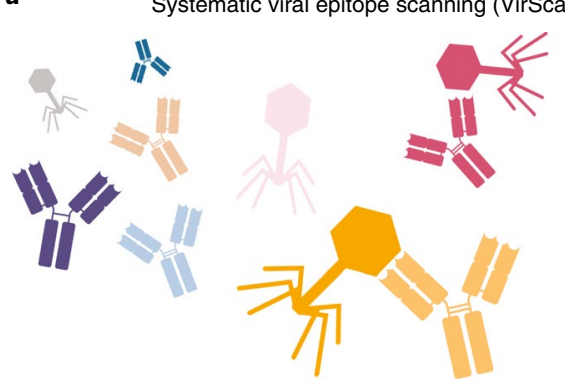

93,904 epitopes (56 amino acids)

1,276 viral strains

206 viral species b

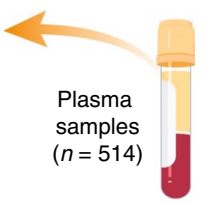

Longitudinal samples early in life

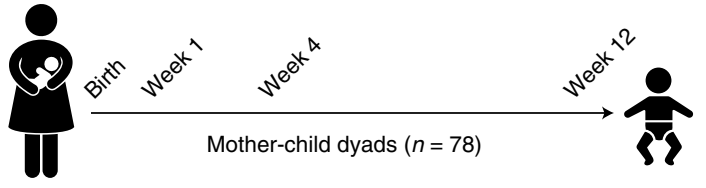

Antiviral antibodies in parents

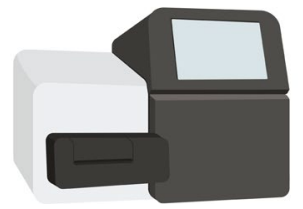

c

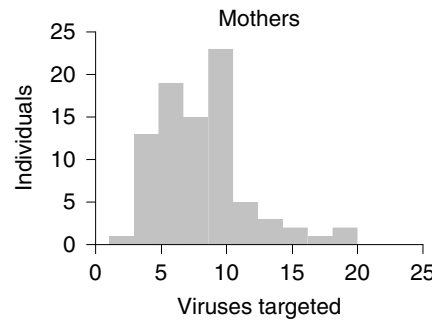

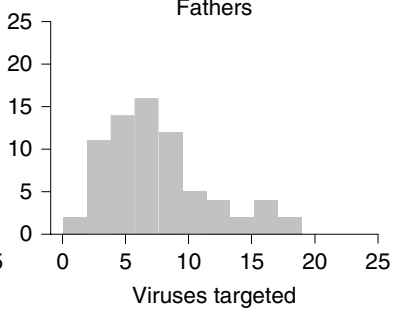

d Most commonly targeted viruses

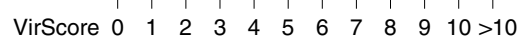

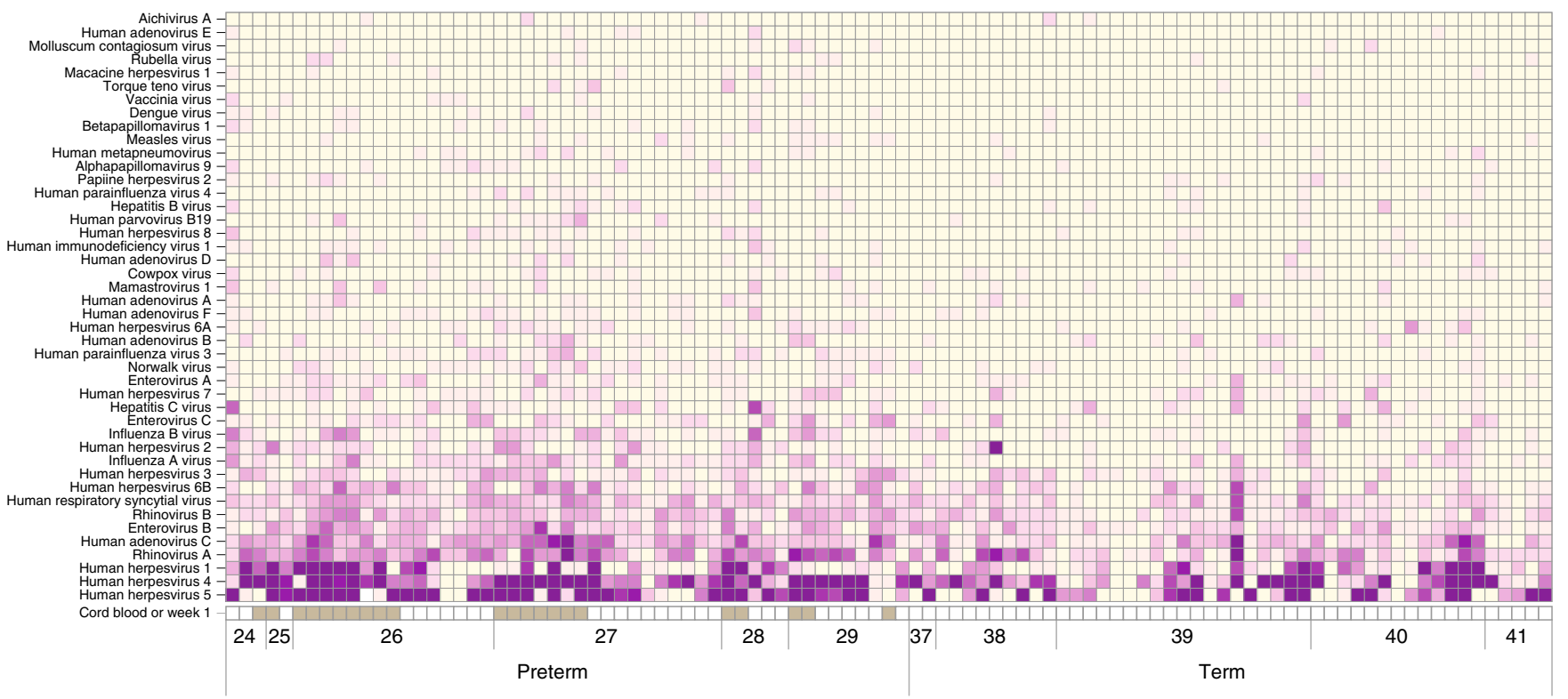

Fig. 1 | Global profiling of maternal antibodies. a, Method overview. b, Sampling diagram and cohort description. c, Number of viruses targeted (VirScores $>1$ ) in paternal and maternal samples collected during week 1 after birth. d, Top 44 viruses most frequently targeted (VirScores $\geq 2$ ) by maternal antibodies in preterm ( $<30$ weeks gestation, $n=32$ ) and term ( $>37$ weeks gestation, $n=46)$ children. The first available sample for each child is included, either at birth (cord blood) or day 2-3 (week 1), as indicated by the color code box at the bottom. The samples are ordered and labeled by their gestational age (weeks) at birth.

children (Fig. 2b). This suggests that the repertoire of antibodies to immunodominant epitopes in newborns mirrors the repertoire of their mothers ${ }^{18}$. The knowledge of such immunodominant epitopes and their variance between children is important for understanding susceptibility to infections; it also has implications for vaccine design. We mapped 10,362 epitopes targeted in any of the samples analyzed and annotated them to their respective viral protein and viral species (Supplementary Table 1). For the 44 most commonly targeted viruses (VirScores $\geq 2$ ), we defined immunodominant epitopes as epitopes targeted by at least $50 \%$ of seropositive children (Fig. 2c). We found differences in the presence of immunodominant peptides among different viruses. For instance, rhinoviruses A and B have multiple epitopes targeted in $>75 \%$ of seropositive children, while influenza A and B have no such immunodominant epitopes (Fig. 2c). One possible interpretation of this is that this is a consequence of strong antigenic shifts among influenza viruses between seasons. CMV, EBV, and enterovirus $\mathrm{B}$ and $\mathrm{C}$ were similar to rhinoviruses in that multiple immunodominant epitopes targeted in the majority of seropositive children could be found (Fig. 2c). The frequencies of immunodominant epitopes were largely comparable between preterm and term children, further suggesting that IgG transfer occurs early during gestation (Extended Data Fig. 3).

RS-virus is an important cause of morbidity and mortality in newborns and young children ${ }^{19}$. We found multiple RS-virus epitopes targeted by $>50 \%$ of seropositive children; all of these involved major surface glycoprotein G (UniProt identification nos. P27021, 


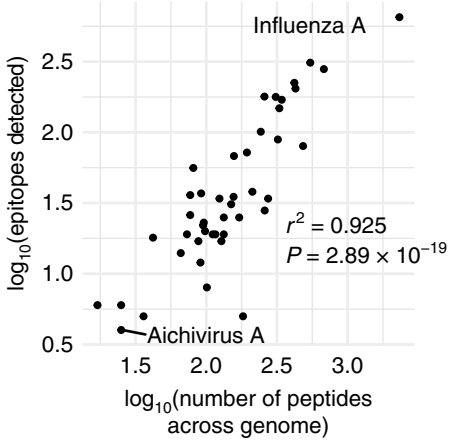

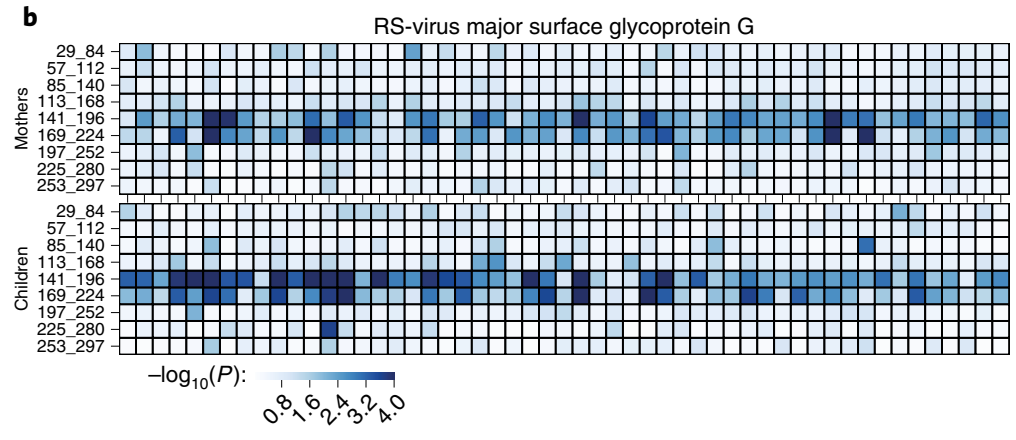

C

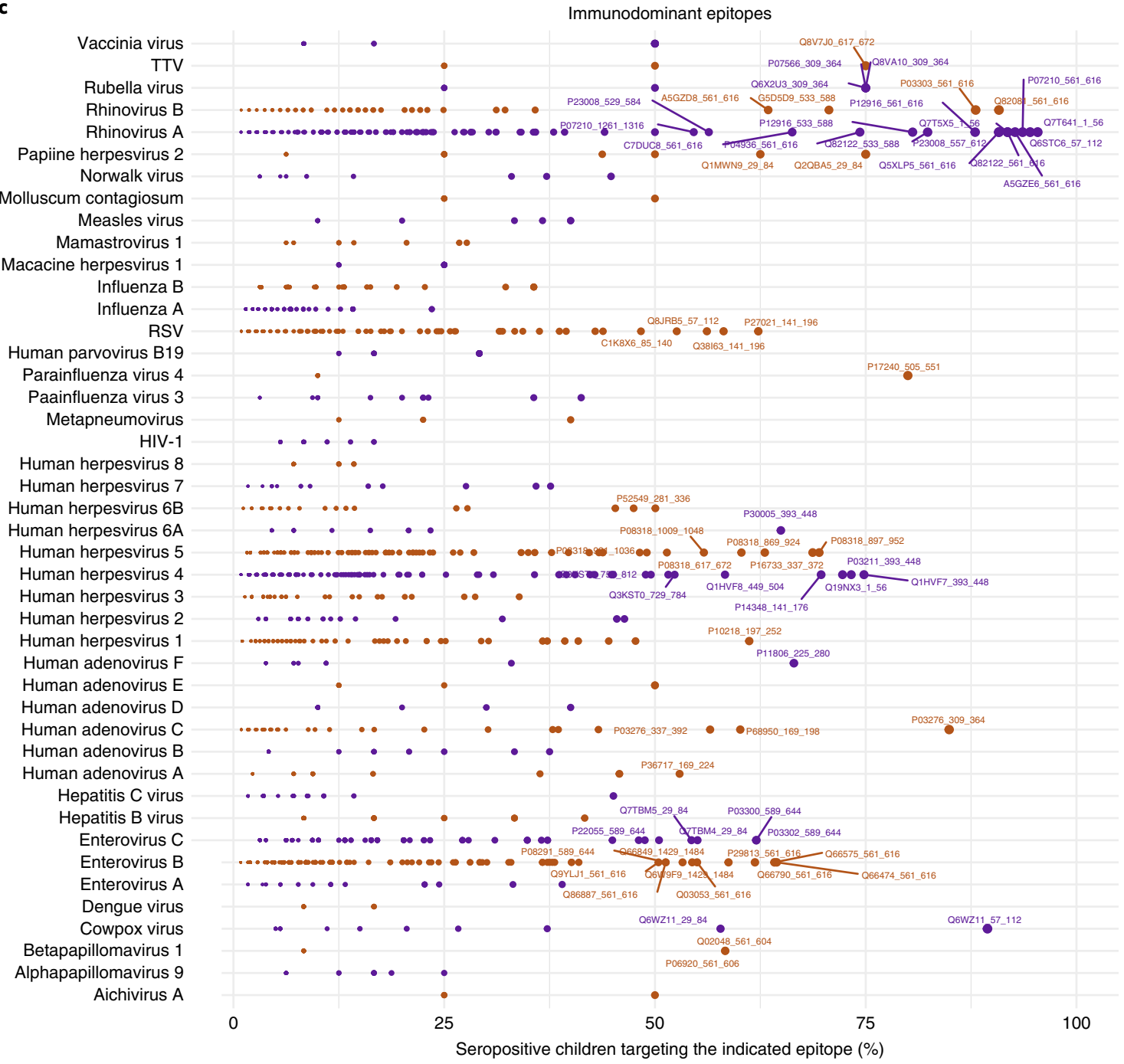

Fig. 2 | Epitopes targeted by maternal antibodies. Analyses based on the same samples as in Fig. 1d, that is, the first sample collected from each child, either at birth (cord blood) or during the first few days of life $(n=78)$. a, Viral genome size versus the number of epitopes targeted by passive immunity. Pearson's correlation $r^{2}$ and statistical test with the null hypothesis $\mathrm{H}_{0}: r^{2}=0$. b. Peptides of RS-virus major surface glycoprotein $\mathrm{G}$ targeted by IgG antibodies in mothers (top) and their children (bottom). The statistical test shows the significance of enrichment $-\log _{10}(P)$. c, For the 44 most frequently targeted viruses in Fig.1d, all epitopes targeted in seropositive children are shown; the most immunodominant epitopes, found in $>50 \%$ of seropositive children labeled with their UniProt identification number and amino acid peptides.

Q38163, Q8JRB5, and C1K8X6). This protein is important for virus attachment and infectivity ${ }^{20}$ (Fig. 2c). For other viruses, only one immunodominant epitope was found. For example, amino acids 505-551 of the parainfluenza virus type 4 nucleoprotein was targeted by $\sim 80 \%$ of seropositive children; no other immunodominant epitopes were found for this virus (Fig. 2c).
We particularly wanted to compare antibody levels among preterm and term children given previous reports of lower levels in preterm children ${ }^{1,2,4}$. Although concentrations of antibodies correlated with the VirScores in VirScan, we wanted to quantify antibody levels further and performed multiplex enzyme-linked immunosorbent assay (ELISA) assays to quantify IgG specific to known 


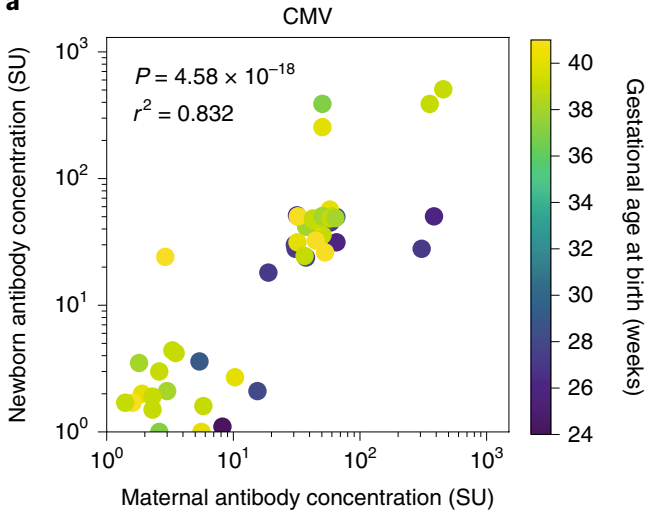

b

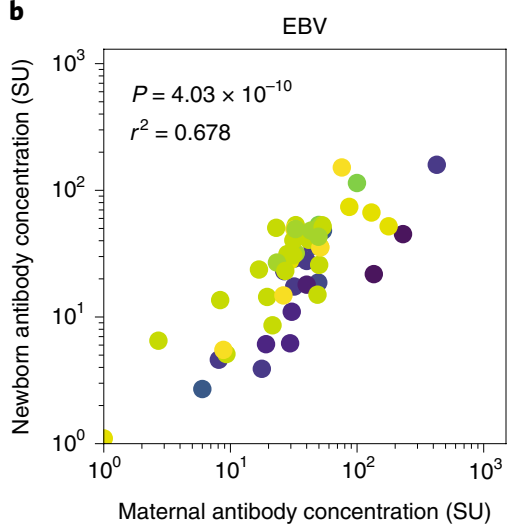

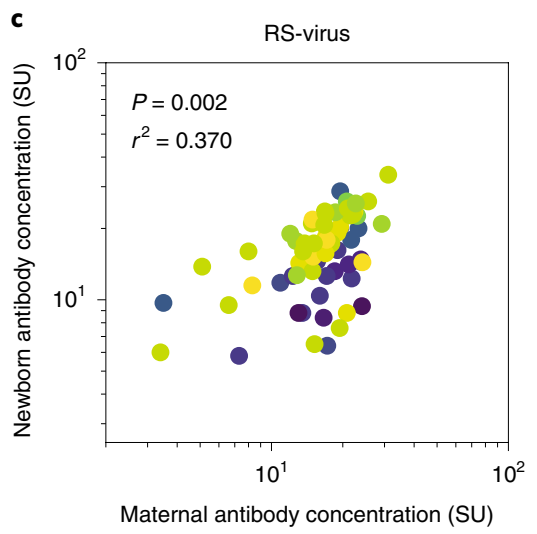

d

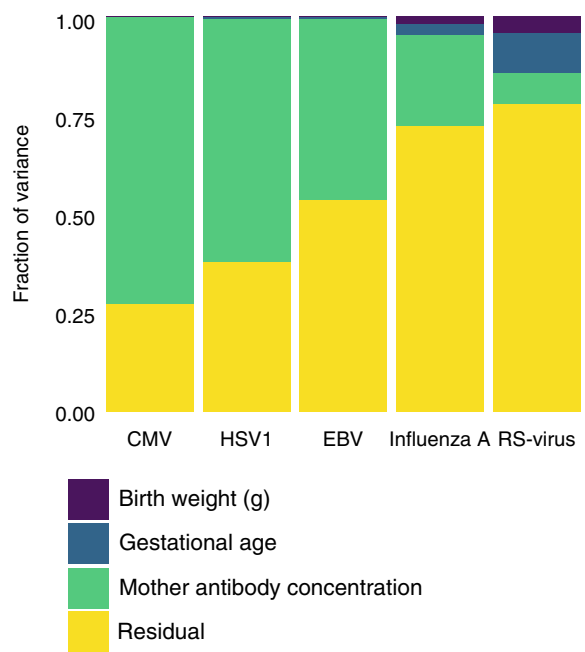

e

\begin{tabular}{rcccccc} 
& \multicolumn{2}{c}{ CMV } & \multicolumn{2}{c}{ EBV } & \multicolumn{2}{c}{ RS-virus } \\
& Preterm & Term & Preterm & Term & Preterm & Term \\
Half-life (d) & 47.8 & 48.0 & 54.9 & 84.5 & 66.3 & 81.6 \\
Antibody presence (d) & 67.4 & 69.3 & 79.1 & 121.9 & 95.3 & 117.8 \\
& \multicolumn{2}{c}{ HSV-1 } & & \multicolumn{2}{c}{ Influenza A } & \\
& \multicolumn{2}{c}{ Preterm } & Term & Preterm & Term & \\
Half-life (d) & 51.8 & 74.3 & 65.4 & 78.0 & \\
Antibody presence (d) & 74.7 & 107.2 & 94.5 & 112.6 &
\end{tabular}

Fig. 3 | Determinants of maternal antibody concentrations. a-c, Relative concentrations of anti-CMV- $(n=64)$, EBV- $(n=64)$, and RS-virus-specific $(n=64)$ antibodies in mothers and their children, respectively. Pearson's correlation $r^{2}$ and statistical test with the null hypothesis $\mathrm{H}_{0}: r^{2}=0$. The points are colored by gestational age at birth to allow comparisons between preterm and term children. $\mathbf{d}$, Fraction of variance in newborn IgG concentration explained (sums of squares) for each of the indicated viruses. e, Estimated half-life and total life span of IgG antibodies to CMV, EBV, RS-virus, HSV-1, and influenza A based on longitudinal ELISA data in preterm and term children.
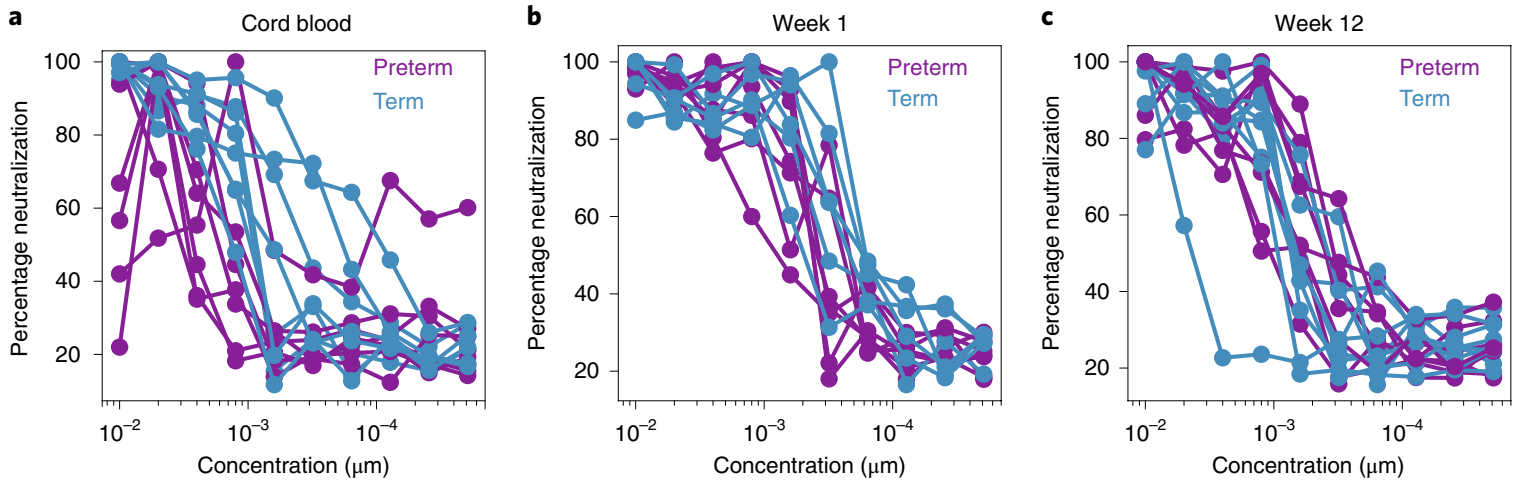

Fig. 4 | Neutralizing capacity of anti-RS-virus antibodies in preterm and term children. a, Dilution curve and percentage neutralizing capacity of maternal antibodies in cord blood (preterm $n=7$, term $n=7$ ) b. Dilution curve and percentage neutralizing capacity of maternal antibodies in week 1 samples (preterm $n=8$, term $n=7$ ). c, Dilution curve and percentage neutralizing capacity of maternal antibodies in week 12 samples (preterm $n=9$, term $n=9$ ).

immunodominant epitopes from five common viruses. Overall, the main determinant of IgG concentration in newborns was maternal IgG concentration, as shown for CMV and EBV (Fig. 3a,b).
RS-virus antibody concentrations were poorly explained by maternal IgG levels, while gestational age was a more influential factor (Fig. 3c) suggesting that different antibodies in the repertoire 
follow different rules of active transfer across the placenta. For both influenza A and RS-virus most of the variance remains unexplained by gestational age, maternal IgG concentration, and birth weight (Fig. 3d). This warrants more research into the possible factors, such as isotype differences, glycosylation patterns, binding capacity to the neonatal $\mathrm{Fc}$ receptor $(\mathrm{FcRn})$, and other possible determinants of IgG transfer ${ }^{21}$.

The life span of maternal antibodies is another important factor for understanding the duration of elevated susceptibility to infection and know when vaccines should be administered to avoid negative interference of maternal antibodies ${ }^{3,22}$. We found that IgG concentrations were generally higher in term versus preterm children, but the rates of decay were comparable leading to longer halflives and overall maternal IgG duration in term children (Fig. 3e). This pattern was similar for all viruses analyzed-CMV, EBV, influenza A, HSV-1, and RS-virus-suggesting a general process of antibody decay.

Given that the repertoires of maternal antiviral IgG were comparable between extremely preterm and term children, albeit with lower IgG concentrations in preterm children, we were interested in evaluating the functional capacity of these antibodies in such different groups of children. We focused on the RS-virus, which is responsible for much morbidity and mortality, particularly in preterm children. By testing the neutralizing capacity of serial dilutions of newborn plasma from RS-virus seropositive children, we assessed this neutralizing capacity at birth, week 1, and week 12 of life using a cytopathogenic effect-based microneutralization assay. We found that cord blood samples overall differed significantly in their ability to neutralize RS-virus in in vitro culture, whereas neutralizing capacity was more homogenous at subsequent time points (Fig. $4 \mathrm{a}-\mathrm{c}$ ). Although there was a trend toward higher neutralizing capacity in the plasma samples from the cord blood of term children compared to preterm children, this difference was not seen for plasma samples from weeks 1 and 12. The neutralizing capacity of maternal IgG targeting the RS-virus was the same for preterm and term children overall (Fig. 4b,c). One possible explanation for this discrepancy between time points is that the strong inflammatory response in the cord blood of extremely preterm children ${ }^{17}$ could possibly impact the neutralizing capacity of the RS-virus. Overall, these findings suggest that extremely preterm children are equally well protected by maternal RS-virus-specific antibodies compared to term children, at least until three months of age. This finding was not exclusive to the RS-virus since plasma samples from preterm and term children also neutralized influenza A, (H3N2/Hong Kong/1968) to a similar extent (Extended Data Fig. 4). This despite having lower concentrations of IgG antibodies specific for these viruses. We hypothesize that the higher mortality of the RS-virus and influenza-virus infection in preterm and term children ${ }^{23}$ is not explained by a lack of passive immunity, albeit possibly a shorter duration of passive immunity due to lower IgG concentration at birth. Other possible explanations could instead be a lower residual lung capacity and a generally lower resilience toward severe illness as a consequence of low birth weight and intensive care.

In summary, we have characterized the global repertoire of maternal IgG antibodies to 93,904 different viral epitopes from 206 different viruses in 78 newborn children during their first three months of life. Our findings show that extremely preterm and term children are equipped with comparable passive immunity to viruses at birth, with repertoires that mirror that of their mothers, and centered on a defined set of immunodominant epitopes we have described. We have also shown that the determinants of antibody concentration differ among viruses, implying that other features of IgG molecules, such as isotypes, posttranslational modifications, and binding to the neonatal FcRn responsible for the transfer of humoral immunity to the fetus, might be of importance and should be investigated further in future studies. These findings also suggest that the elevated risk of infection in preterm over term newborns is not determined by a lack of maternal antibodies; instead, it might be explained by weaker physical barriers in the skin, intestine, and lung or differences in exposure due to intensive care, intravenous catheters, and breathing tubes.

\section{Online content}

Any methods, additional references, Nature Research reporting summaries, source data, statements of data availability and associated accession codes are available at https://doi.org/10.1038/ s41591-019-0392-8.

Received: 15 September 2018; Accepted: 8 February 2019; Published online: 18 March 2019

\section{References}

1. Hobbs, J. R. \& Davis, J. A. Serum $\gamma$ G-globulin levels and gestational age in premature babies. Lancet 289, 757-759 (1967).

2. Conway, S. P., Dear, P. R. \& Smith, I. Immunoglobulin profile of the preterm baby. Arch. Dis. Child. 60, 208-212 (1985).

3. Siegrist, C.-A. \& Aspinall, R. B-cell responses to vaccination at the extremes of age. Nat. Rev. Immunol. 9, 185-194 (2009).

4. Salimonu, L. S., Ladipo, O. A., Adeniran, S. O. \& Osukoya, B. O. Serum immunoglobulin levels in normal, premature and postmature newborns and their mothers. Int. J. Gynaecol. Obstet. 16, 119-123 (1978).

5. Leuridan, E., Hens, N., Hutse, V., Aerts, M. \& Van Damme, P. Kinetics of maternal antibodies against rubella and varicella in infants. Vaccine 29, 2222-2226 (2011)

6. van der Zwet, W. C., Vandenbroucke-Grauls, C. M. J. E., van Elburg, R. M., Cranendonk, A. \& Zaaijer, H. L. Neonatal antibody titers against varicellazoster virus in relation to gestational age, birth weight, and maternal titer. Pediatrics 109, 79-85 (2002).

7. Ochola, R. et al. The level and duration of RSV-Specific maternal IgG in infants in Kilifi Kenya. PLoS ONE 4, e8088 (2009).

8. Francis, J. P. et al. Maternal antibodies to pneumolysin but not to pneumococcal surface protein A delay early pneumococcal carriage in high-risk Papua New Guinean infants. Clin. Vaccine Immunol. 16, 1633-1638 (2009).

9. Leuridan, E. et al. Early waning of maternal measles antibodies in era of measles elimination: longitudinal study. BMJ 340, c1626 (2010).

10. Watanaveeradej, V. et al. Transplacentally transferred maternal-infant antibodies to dengue virus. Am. J. Trop. Med. Hyg. 69, 123-128 (2003).

11. Jiang, Y. et al. Maternal antiviral immunoglobulin accumulates in neural tissue of neonates to prevent HSV neurological disease. MBio 8, e00678-17 (2017).

12. Aizawa, Y. et al. Role of maternal antibodies in infants with severe diseases related to human parechovirus type 31. Emerg. Infect. Dis. 21, 1966-1972 (2015)

13. Winkelstein, J. A. et al. X-linked agammaglobulinemia: report on a United States registry of 201 patients. Medicine (Baltimore) 85, 193-202 (2006)

14. Hernandez-Trujillo, V. P. et al. Autoimmunity and inflammation in X-linked agammaglobulinemia. J. Clin. Immunol. 34, 627-632 (2014).

15. Jennewein, M. F., Abu-Raya, B., Jiang, Y., Alter, G. \& Marchant, A. Transfer of maternal immunity and programming of the newborn immune system. Semin. Immunopathol. 39, 605-613 (2017).

16. Xu, G. J. et al. Comprehensive serological profiling of human populations using a synthetic human virome. Science 348, aaa0698 (2015)

17. Olin, A. et al. Stereotypic immune system development in newborn children. Cell 174, 1277-1292.e14 (2018).

18. Lozano, N. A. et al. Expression of FcRn receptor in placental tissue and its relationship with IgG levels in term and preterm newborns. Am. J. Reprod. Immunol. 80, e12972 (2018).

19. Scheltema, N. M. et al. Global respiratory syncytial virus-associated mortality in young children (RSV GOLD): a retrospective case series. Lancet Glob. Health 5, e984-e991 (2017).

20. Feldman, S. A., Audet, S. \& Beeler, J. A. The fusion glycoprotein of human respiratory syncytial virus facilitates virus attachment and infectivity via an interaction with cellular heparan sulfate. J. Virol. 74, 6442-6447 (2000).

21. Wilcox, C. R., Holder, B. \& Jones, C. E. Factors affecting the FcRn-mediated transplacental transfer of antibodies and implications for vaccination in pregnancy. Front. Immunol. 8, 1294 (2017).

22. Jennewein, M. F. \& Alter, G. The immunoregulatory roles of antibody glycosylation. Trends Immunol. 38, 358-372 (2017).

23. Resch, B., Kurath, S. \& Manzoni, P. Epidemiology of respiratory syncytial virus infection in preterm infants. Open Microbiol. J. 5, 135-143 (2011) 


\section{Acknowledgements}

We are grateful to all the families taking part in this study, all colleagues at the Karolinska University Hospital neonatology and delivery wards. We are also indebted to S. Elledge and T. Kula at Harvard University for sharing the VirScan bacteriophage library and their assistance. The study was funded by a European Research Council starting grant (no. ERC-StG-677559), Wallenberg Clinical Fellow grant (no. MMW 2017.0127), Karolinska Institutet (KI Research Assistant 2015 grant), and the Swedish Research council (grant no. 2015-03028) to P.B.

\section{Author contributions}

C.P. established the VirScan method and performed all the experimental analyses using VirScan and ELISA. D.N. and P.B. performed the computational analyses. E.H., A.K.B., P.B., A.G., and K.B. enrolled the children and collected the samples. A.O., T.L., J.M., and J.W. assisted with sample organization, processing, and metadata analysis. Y.C. developed the data analysis infrastructure and database. P.B., E.H., and K.B. designed the study. P.B., D.N., and C.P. created the figures and wrote the manuscript with input from all coauthors.

\section{Competing interests}

The authors declare no competing interests.

\section{Additional information}

Extended data is available for this paper at https://doi.org/10.1038/s41591-019-0392-8.

Supplementary information is available for this paper at https://doi.org/10.1038/ s41591-019-0392-8.

Reprints and permissions information is available at www.nature.com/reprints. Correspondence and requests for materials should be addressed to P.B.

Publisher's note: Springer Nature remains neutral with regard to jurisdictional claims in published maps and institutional affiliations.

(C) The Author(s), under exclusive licence to Springer Nature America, Inc. 2019 


\section{Methods}

Study design. Plasma samples were longitudinally collected from 32 preterm and 46 term children at the Karolinska University Hospital at delivery (from umbilical cord) and during weeks 1,4 , and 12 (from peripheral blood). Plasma was also collected from the peripheral blood of mothers around one week after the time of delivery.

Ethics statement. The parents of all children participating in the study received oral and written information about the study. Informed consent was obtained before any analyses. The study protocol was approved by the Stockholm regional ethical review board, with case numbers 2014/921-32 and 2016/512-31/1. The study protocol follows the Declaration of Helsinki (DoH/Oct2008) and the data collection procedures are in accordance with the European Union General Data Protection Regulation.

VirScan. A global analysis of the maternal antiviral immunoglobulin $\mathrm{G}$ repertoire in the individual serum samples was performed as in Xu et al. ${ }^{16}$. Briefly, the assay consisted of an immunoprecipitation of antibody-targeted bacteriophages from a library displaying proteome-wide peptides from all viruses known to infect human cells, except the Zika Virus (206 species and 1,276 strains). After immunoprecipitation, antibody-targeted bacteriophages underwent massive parallel DNA sequencing to identify the targeted peptide. The bacteriophage library was kindly shared by the $\mathrm{S}$. Elledge's group and expanded in our laboratory at Science for Life Laboratory (SciLifeLab), Karolinska Institutet, Stockholm, Sweden. Five rounds of expansion (approximately 25 petri dishes each) were carried out to collect the supernatants. This minimized the possibility of bacteriophage overexpansion and preserved the frequency of each bacteriophage in the original library. The final bacteriophage library (pooled) was used to determine plaque-forming units and underwent lysis and Illumina sequencing to also assess phage composition (input). Moreover, the phage library was directly incubated with beads alone (no IgG) to identify unspecific binding during the capture of immunocomplexes (beads). Epitopes not detected during input (phages not successfully grown during expansion) or found in beads were not considered during the data analysis. Illumina sequencing was performed by the National Genomics Infrastructure, SciLifeLab (HiSeq Rapid Mode single read $1 \times 50$ base pairs (bp)).

After sequencing, we used Bowtie to map the sequencing reads to the original library sequences and SAMtools to count the number of reads for each peptide during input as well as in each sample output. Given that a large number of peptides are not enriched, the observed distribution of output read counts was used as a null distribution. A zero-inflated generalized Poisson distribution was found to fit well the frequency of each peptide in the sample output. Subsequently, we fitted a zero-inflated generalized Poisson model to the distribution of output counts and regressed the parameters at a particular input read count. We used this model to calculate $a-\log _{10}(P)$ for the probability of enrichment for each peptide. A reproducibility threshold of 2.3 in both technical sample replicates was used to determine significantly enriched peptides. Then, peptides from a virus were grouped and epitopes that were explained as cross-reactions from another virus, as well as from unspecific binding, were removed. The remaining number of unique peptides found defined a VirScore for antibodies to each virus in each sample.

Definition of VirScores from epitope hits. To filter out spurious hits caused by cross-reactive antibodies, we calculated the total number of epitope hits per virus and sorted these in descending order. For each virus in this order, we iterated through all epitope hits and removed hits that shared $>7$ amino acid sequences with any previously observed hit in any of the viruses from the same sample. The remaining hits were considered specific and summed into a VirScore for the virus.

Assessing specificities of antiviral antibodies found in mother-child dyads. To assess the specificities of maternal antiviral antibodies, we retained overlapping viral species in mother-child dyads. We detected antibodies to 134 viral species in the whole cohort (Fig. 1d). Based on very low antibody titers, we determined a threshold of strong protection. Finally, we called an antibody frequently found if the viral species to that antibody was detected with a VirScore $>1$.

Determination of antibody levels in children and adults. Total IgG was measured with an ELISA assay in all longitudinal serum samples from our birth cohort (Human IgG ELISA Kit; catalog no. RAB0001, Sigma-Aldrich). Besides total IgG, semiquantitative ELISA assays for well-known immunodominant epitopes from the following viruses were also measured (all sourced from Abcam): CMV (Human Anti-Cytomegalovirus IgG ELISA Kit (CMV); catalog no. ab108724); RS-virus (Human Anti-Respiratory syncytial virus IgG ELISA Kit (RSV); catalog no. ab108765); HSV-1 (Human Anti-Herpes simplex virus Type 1 IgG ELISA Kit; catalog no. ab108737); influenza type A (Human Anti-Influenza virus A IgG ELISA Kit; catalog no. ab108745); and EBV (Human Anti-Epstein-Barr virus IgG ELISA Kit (EBV-EBNA); catalog no. ab108731). Results are shown as standard units (SU).

Serological analysis: statistical model of antibody kinetics. To capture the kinetics of passively acquired maternal antibodies against CMV, EBV, HSV1 , influenza A, and RS-virus, a stochastic model was adopted from ref. ${ }^{24}$. For each individual, log-transformed levels of antibodies $x$, at a particular age $a$, are described by a normal distribution with age-dependent mean $m(a)$ and s.d. $s$ :

$$
x \approx N\left(m(a), s^{2}\right)
$$

The log-transformed levels of maternal antibodies decay approximately linearly with age and with a decay rate, $d$, from $\ln \left(m_{\mathrm{o}}+b\right)$ for low ages (first days of life), and approaches a detectable level of antibody, termed the baseline level, $b$, for higher ages. The mean log-transformed level of maternal antiviral antibodies varies with age.

$$
m(a)=\ln \left(m_{\mathrm{o}} \mathrm{e}^{-d a}+b\right)
$$

We used the maximum likelihood estimation to regress the parameters $m, b, d$, and $s$ to fit the age-specific levels of antibodies for preterm and term children. A Markov chain Monte Carlo simulation was used to quantify the uncertainty of these parameter estimates. We also calculated the half-time $(\ln (2) / d)$ and mean-life $(1 / d)$ of antibodies using the same estimated parameters.

Evaluation of the neutralizing activity against the RS-virus. Neutralizing activity against the RS-virus was tested using plasma from RS-virus seropositive children (ELISA-verified). Cytopathogenic effect-based microneutralization in human epithelial type 2 cells was performed by Integrated BioTherapeutics.

Reporting Summary. Further information on research design is available in the Nature Research Reporting Summary linked to this article.

\section{Code availability}

The pipeline for processing the VirScan data has been published previously; the script for all other analyses described in the paper is available from https://github. com/Brodinlab/maternal_abs.

\section{Data availability}

All VirScan sequencing data (Figs. 1 and 2) is deposited at the NCBI Sequence Read Archive database (PRJNA516865). The ELISA (Fig. 3) and virus neutralization raw data (Fig. 4) are readily available upon request.

\section{References}

24. van den Hof, S., Wallinga, J., Widdowson, M. A. \& Conyn-van Spaendonck, M. A. Protecting the vaccinating population in the face of a measles epidemic: assessing the impact of adjusted vaccination schedules. Epidemiol. Infect. 128, 47-57 (2002). 
a
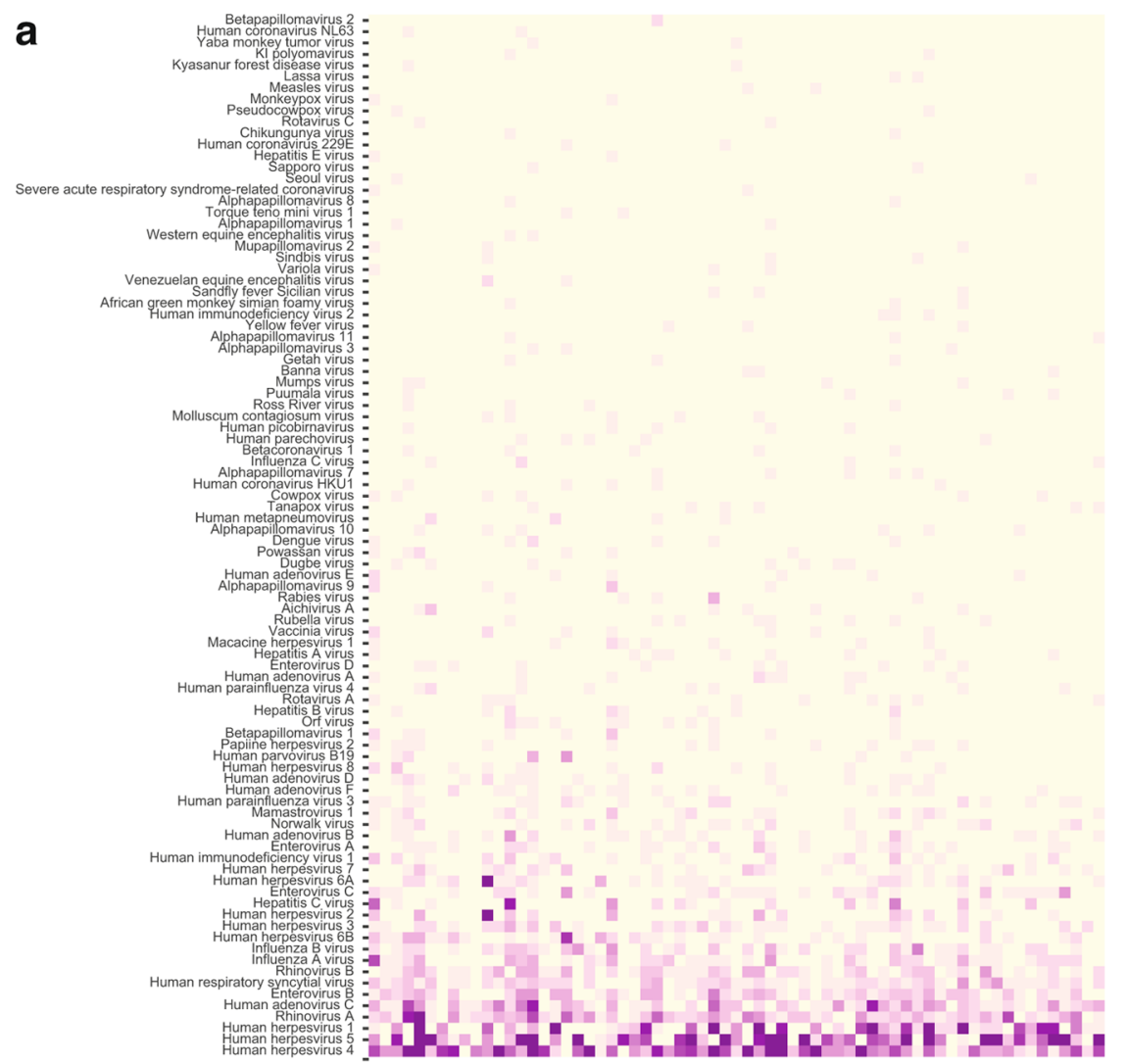

b
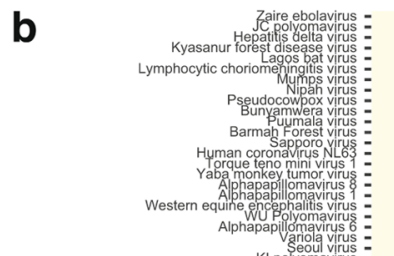

\section{VirScore

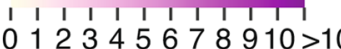

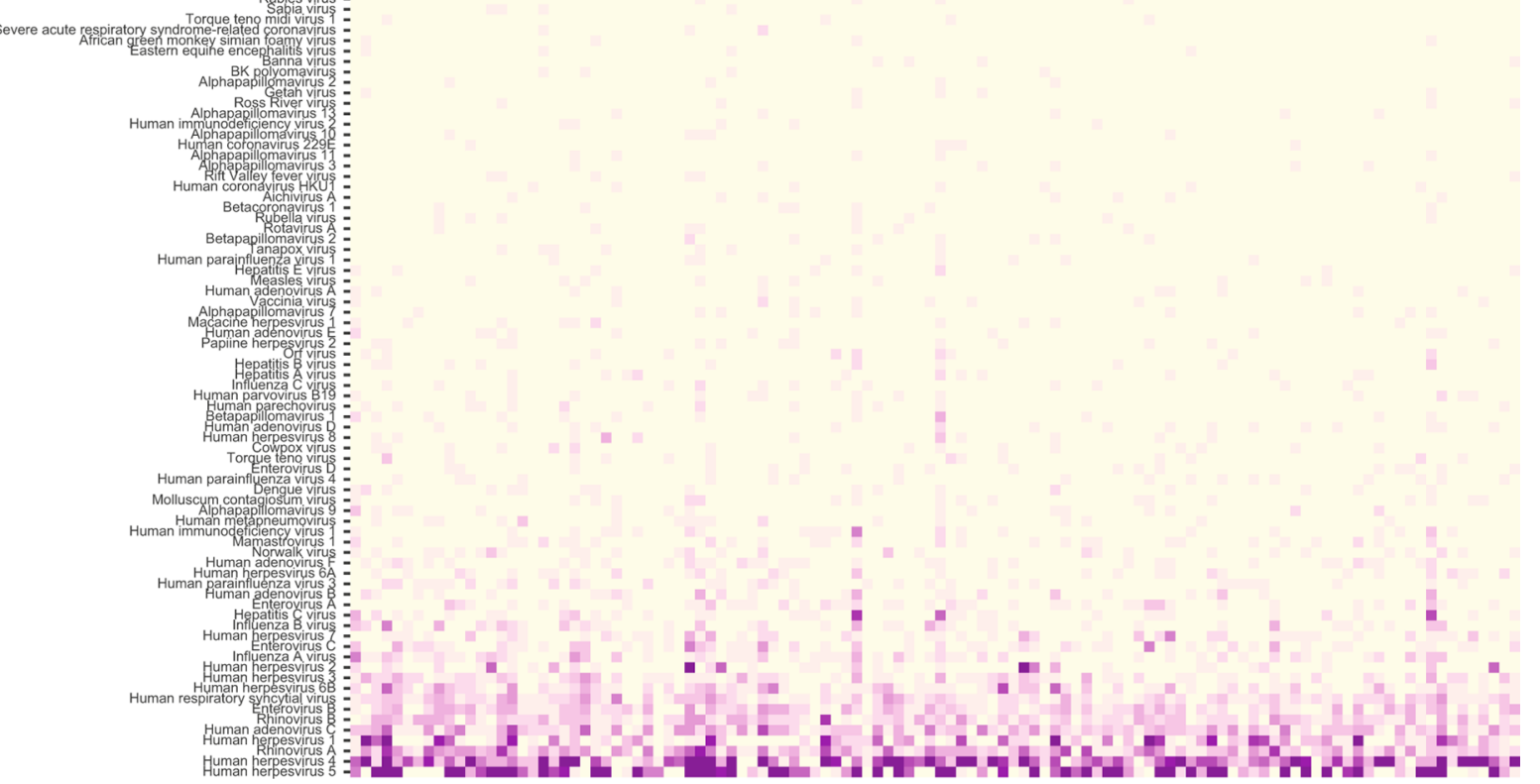

Extended Data Fig. 1| Parental repertoires of antiviral antibodies. a, Viruses targeted by antibodies in fathers. b, Viruses targeted by antibodies in mothers. 


\section{Preterm/Term maternal IgG repertoires}

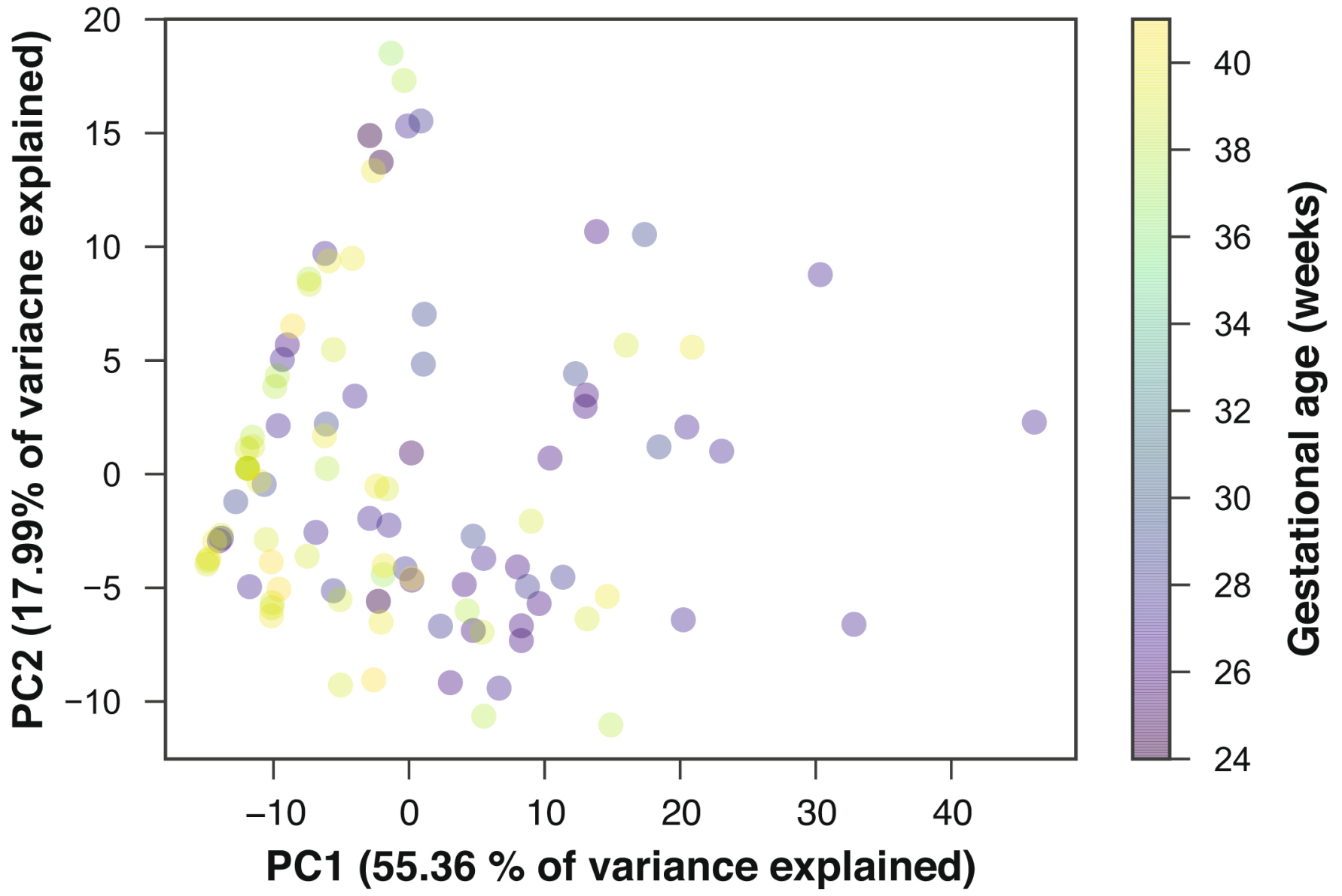

Extended Data Fig. 2 | Preterm/term repertoires of antiviral antibodies. Principal component analysis based of global antiviral repertoires (species level). Each dot represents a unique individual child $(n=102)$; the first sample available at birth or first days of life is used as in Fig. 1d and the dots are colored by gestational age at birth for each child. 


\section{Immunodominant epitopes in preterm and term children}

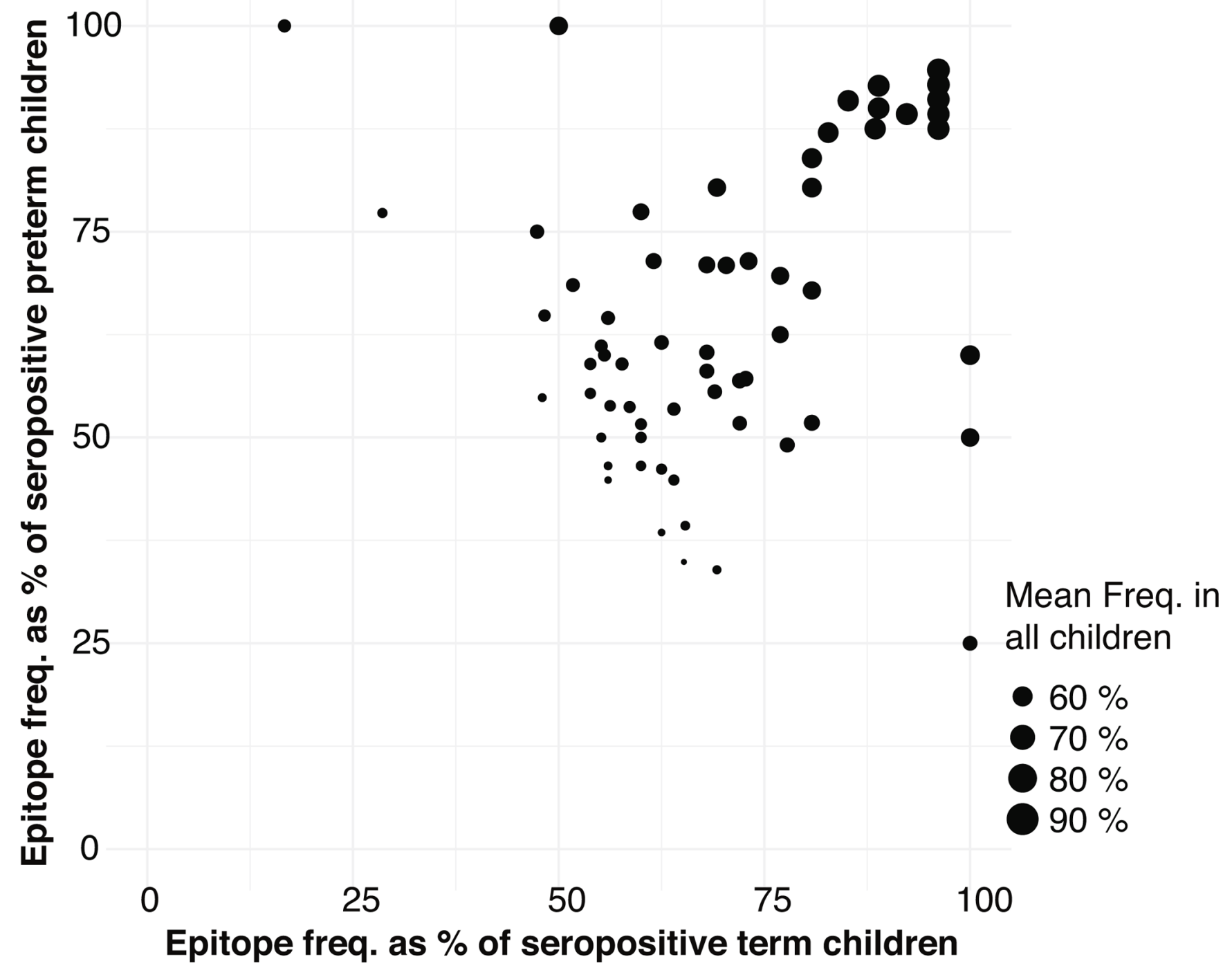

Extended Data Fig. 3 | Antibodies to immunodominant epitopes in preterm and term children. The frequencies of maternal lgG to 70 immunodominant epitopes (mean frequency $>50 \%$ of seropositive children) in term ( $x$ axis) and preterm ( $y$ axis) children. The point size represents the mean frequencies in all children. 


\section{Inf-A (H3N2, Hong Kong/1968) neutralization}

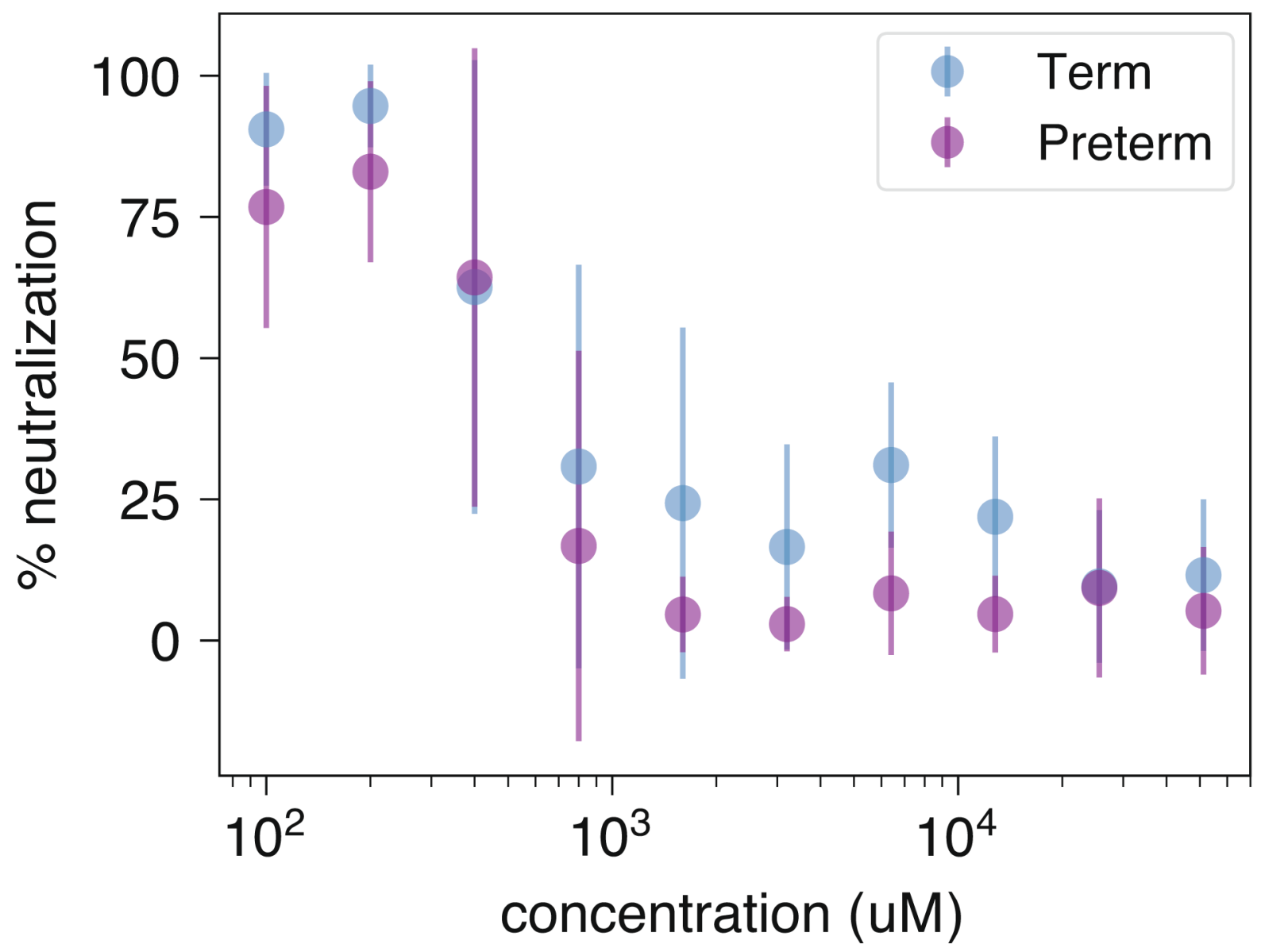

Extended Data Fig. 4 | Neutralizing capacity of anti-influenza A antibodies in preterm and term children. Dilution curve and percentage neutralizing capacity of maternal antibodies in cord blood (preterm $n=13$, term $n=10$ ). The points indicate the mean values and the error bars define the minimum and maximum values of viral neutralization. 


\section{Reporting Summary}

Nature Research wishes to improve the reproducibility of the work that we publish. This form provides structure for consistency and transparency in reporting. For further information on Nature Research policies, see Authors \& Referees and the Editorial Policy Checklist.

\section{Statistical parameters}

When statistical analyses are reported, confirm that the following items are present in the relevant location (e.g. figure legend, table legend, main text, or Methods section).

$\mathrm{n} / \mathrm{a} \mid$ Confirmed

\The exact sample size $(n)$ for each experimental group/condition, given as a discrete number and unit of measurement

$\square$ An indication of whether measurements were taken from distinct samples or whether the same sample was measured repeatedly

The statistical test(s) used AND whether they are one- or two-sided

Only common tests should be described solely by name; describe more complex techniques in the Methods section.

$\bigotimes$ A description of all covariates tested

$\bigotimes$ A description of any assumptions or corrections, such as tests of normality and adjustment for multiple comparisons

A full description of the statistics including central tendency (e.g. means) or other basic estimates (e.g. regression coefficient) AND

variation (e.g. standard deviation) or associated estimates of uncertainty (e.g. confidence intervals)

$\varnothing$ For null hypothesis testing, the test statistic (e.g. $F, t, r$ ) with confidence intervals, effect sizes, degrees of freedom and $P$ value noted

Give P values as exact values whenever suitable.

Х $\square$ For Bayesian analysis, information on the choice of priors and Markov chain Monte Carlo settings

Х $\square$ For hierarchical and complex designs, identification of the appropriate level for tests and full reporting of outcomes

Х $\square$ Estimates of effect sizes (e.g. Cohen's $d$, Pearson's $r$ ), indicating how they were calculated

$\varnothing$ Clearly defined error bars

State explicitly what error bars represent (e.g. SD, SE, CI)

Our web collection on statistics for biologists may be useful.

\section{Software and code}

Policy information about availability of computer code

Data collection $\quad$ For processing sequencing data, scripts written in Python version 3.5.4, Bowtie version 1.0.2 and package samtools version 1.1 was used for reads alignment. For downstream analysis and plotting numpy version 1.11.3, pandas version 0.21.0, and matplotlib version 2.1.2 was used.

Data analysis

we used VirScan pipeline as per (Xu et al. (2015)) and our own python scripts. All code is available: https://github.com/Brodinlab/ maternal_abs

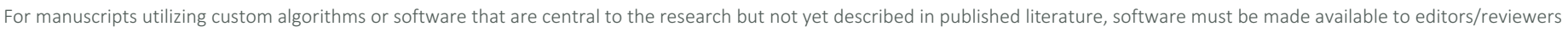

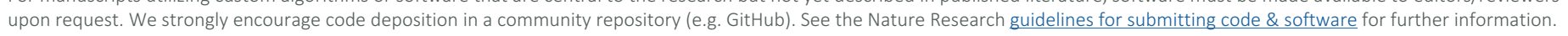


Policy information about availability of data

All manuscripts must include a data availability statement. This statement should provide the following information, where applicable:

- Accession codes, unique identifiers, or web links for publicly available datasets

- A list of figures that have associated raw data

- A description of any restrictions on data availability

VirScan sequencing data is deposited at the NCBI SRA database with BioProject ID: PRJNA516865 (https://www.ncbi.nlm.nih.gov/bioproject/PRJNA516865). ELISA and Virus neutralization data is available upon request.

\section{Field-specific reporting}

Please select the best fit for your research. If you are not sure, read the appropriate sections before making your selection.

$\bigotimes$ Life sciences $\quad \square$ Behavioural \& social sciences $\quad \square$ Ecological, evolutionary \& environmental sciences

For a reference copy of the document with all sections, see nature.com/authors/policies/ReportingSummary-flat.pdf

\section{Life sciences study design}

All studies must disclose on these points even when the disclosure is negative.

Sample size In total, 514 plasma samples were included. Plasma samples were longitudinally collected from 32 preterm and 46 fullterm children (78 from umbilical cord and 114, 55 and 80 from weeks 1, 4 and 12, respectively). Besides babies, 72, and 115 plasma samples from fathers and mothers respectively, were also collected after delivery. The number of children included was not based on any power analysis. All children fulfilling inclusion criteria and consenting to participate was included in these analyses.

Data exclusions No data exclusions carried out.

Replication $\quad$ All attempts at replication were successful. All antibody measurements with ELISA were performed in duplicate. Duplicates were also analyzed in VirScan.

Randomization n Samples were randomized before VirScan and ELISA analysis.

Blinding Investigators were blinded to sample origin. Serum samples were already sent to the laboratory with a Sample id, making no possible to associate between Sample_group or Subject_id during the experimental process. All samples involving the same family were processed together. Only during analysis were samples linked to individuals.

\section{Reporting for specific materials, systems and methods}

Materials \& experimental systems

\begin{tabular}{l|l}
\hline Ia & Involved in the study \\
\hline & $\square$ Unique biological materials \\
$\square$ Antibodies
\end{tabular}

Methods

n/a Involved in the study

X ChIP-seq

Х $\square$ Flow cytometry

Х $\square$ MRI-based neuroimaging

\section{Unique biological materials}

Policy information about availability of materials

Obtaining unique materials The VirScan phage display library is available upon request to professor Stephen Elledge, Harvard. 
Policy information about cell lines

Cell line source(s)

ATCC \#CCL-23

Authentication

ATCC product documentation: https://www.atcc.org/products/all/CCL-23.aspx\#documentation

Mycoplasma contamination

The cell line was tested negative for mycoplasma by PCR. This is performed by the CRO Integrated Biotherapeutics Inc.

Commonly misidentified lines

(See $\underline{I C L A C}$ register)

None

\section{Human research participants}

Policy information about studies involving human research participants

Population characteristics

Newborn children enrolled in the study and their parents. The cohort includes very preterm children, born prior to week 30 gestation, as well as a term group of children born $>37$ weeks of gestation. All children are born in Stockholm, Sweden and followed at Karolinska University Hospital.

Recruitment

All children born at the Karolinska University Hospital were eligible for enrollment except children born with major organ malformation or known chromosomal defects or other significant congenital conditions. Also children unable to take part in return follow-up visits are not eligible for enrollment. The study was approved by local Stockholm ethics board (ID 2014-921/32). 